\title{
An Assessment of the Effects of Laser Energy Deposition for Cavity Flows
}

\author{
Ibrahim Yilmaz and Selin Aradag
}

\begin{abstract}
Laser energy deposition method is a new and promising method as a flow control technique. In this study, this method is applied to three dimensional, turbulent, supersonic cavity flow which is a critical flow control problem. The application of the laser energy deposition is modeled by using a Gaussian temperature profile assuming the density within the spot is initially uniform. The effects of frequency, location and amount of the laser energy are investigated. At low frequency values, negligible reductions for sound pressure levels (SPL) are obtained. As the frequency is increased, SPL reductions up to $3 \mathrm{~dB}$ occur.
\end{abstract}

Index Terms-Active flow control, open cavity, supersonic flow, laser energy deposition.

\section{INTRODUCTION}

The cavity flow mechanism includes complex and unsteady flow fields. Due to these complex flow fields, dangerous interactions may occur in the cavity flow which can cause harmful incidents in their application. Especially in aeronautics applications, the cavity flow configurations are used for the design of interior storages of air vehicles which provides some advantages such as, increase in the lift or decrease in the drag. However, they can decrease the chance of success of aircrafts in their mission because of the hazardous structural interactions in the flow field [1].To eliminate the undesirable impacts, several cavity flow control studies are performed including the suppression of the high sound pressure values and the pressure oscillations in the cavities .

Various flow control techniques are developed as reported in literature. As a result of these studies, it is concluded that active control techniques are more advantageous than the passive ones. They can adapt to different flow conditions and in contrast to the passive ones, they include a small control mechanism which is an important specification for local flow control problems [2]. These techniques are mainly applied by providing an external energy to the system. There are many types of active control techniques such as, mass injection, mechanical excitation, acoustics driving and microjets [3]. A decrease may occur in the efficiency of these techniques due to the installation difficulties and the problems about the reliability of systems, which makes the researchers intend to

Manuscript received November 30, 2012; revised February 2, 2013. The authors would like to acknowledge the funding source, Turkish Scientific and Research Council (TUBITAK) under grant number 110M539.

I. Yilmaz and S. Aradag are with a TOBB University of Economics and Technology, Ankara, 06560, Turkey (e-mail: iyilmaz@etu.edu.tr; saradag@etu.edu.tr) study different control techniques such as energy deposition methods. Energy deposition is provided by energy sources such as plasma arcs, microwaves and lasers. The advantages of these techniques are that they can be controlled electronically and they can adapt to different conditions rapidly [4].

Since the discovery of the laser induced spark in 1963 , laser beam has been used as an energy deposition method [5]. Schülelin et al. [6], studied laser energy deposition as a flow control technique. The impact of laser energy deposition on the shock wave transformation is investigated. With the increase in the amount of energy, the interactions increase. In the study of Zaidi et al. [7], the impacts of laser energy deposition on shock waves in supersonic flow are examined by using a small scale wind tunnel. By using the results of the interactions between the hot region and the model shock obtained from a small scale wind tunnel, a numerical model is validated for which the energy deposition for practical applications can be optimized. Adelgren et al. [8] studied two standard supersonic flows with laser energy deposition; a sonic transverse injected wall jet and shock waves in a dual domain interactive space in a supersonic turbulent boundary layer. The purpose is to obtain useful changes on the flow properties with laser energy deposition for both cases. Yan et al. [9] studied the effects of laser energy deposition on quiescent air and intersecting shocks experimentally and numerically. The effect of the laser pulse in quiescent air is a formation of an age drop. After a suitable amount of time, this formation turns to a spherical form. This formation triggered the idea of occurrence of a spherically symmetric temperature profile which depends on laser energy deposition with constant volume and they produced a mathematical model of the laser pulse.

In the study of Aradag et al. [10], laser energy deposition is used to control cavity flow oscillations. An open cavity with an L/D ratio of 5.07 and Mach number of 1.5 is investigated. The results indicated that about 5-6 dB reduction in SPL values are observed. Lazar et al. [3] studied energy deposition to control cavity flow, experimentally. With Schlieren imaging and two-component measurements, a desired huge-scaled structure is obtained in the shear layer with laser energy deposition. The studies show that laser energy has a potential on flow control process.

The aim of this study is to observe the impacts of laser energy deposition on pressure fluctuations and sound pressure levels that occur in an open supersonic cavity flow by examining relevant parameters such as amount, 
frequency and location of laser energy deposition.

\section{Statement of the Problem}

The problem includes a cavity configuration with an L/D ratio of 5.07 . The length is $0.12065 \mathrm{~m}$ and the depth is $0.0238 \mathrm{~m}$. The cavity configuration is the same with the one in the study of Ayli et al.[11].

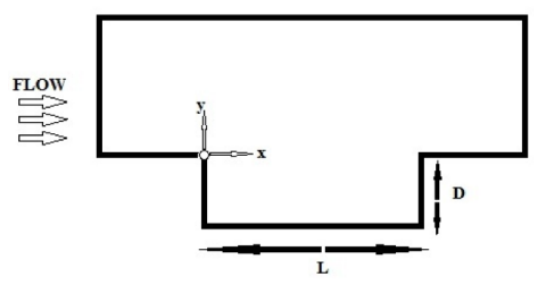

Fig. 1. Cavity configuration

A flow with a Mach number of 1.5 and a Reynolds number of $1.09 \times 10^{6}$ based on the free stream conditions and cavity length is studied. The free stream static temperature is $218 \mathrm{~K}$ and the free stream total pressure is $66.4 \mathrm{kPa}$. [11]

\section{METHODOLOGY}

The computational fluid dynamics simulations are based on one of the cases of the study of Ayli [12]. In the numerical study, $\mathrm{k}-\omega$ turbulence model is utilized. The computations are second order accurate in time and space. Two-dimensional Navier-Stokes equations are solved [12].

The laser energy deposition to the cavity is performed numerically. The numerical model of laser pulse which is obtained in the study of Yan et al. [9] is used. In their study, they observed a spherical plasma as a result of laser energy pulse. They added energy at constant volume and the gas is accepted as an ideal gas. This spherical plasma includes a temperature distribution at the laser deposited region and this temperature distribution is modeled by using Gaussian temperature distribution profile;

$$
\Delta T=\Delta T_{0} e^{\frac{-r^{2}}{\mathrm{r}_{0}^{2}}}
$$

$\Delta \mathrm{T}_{0}$ refers to the maximum temperature difference that occurs at the region where laser pulse is given. This peak temperature difference is calculated by the total amount of laser energy value $\mathrm{E}$, which is given to the system;

$$
E=\int_{0}^{2 \pi} \int_{0}^{\pi} \int_{0}^{\infty} r^{2} \sin \theta \rho_{\infty} c_{v} \Delta \operatorname{Td} \operatorname{dr} \theta \mathrm{d} \emptyset
$$

$\mathrm{c}_{\mathrm{v}}$ is the specific heat ratio at constant volume. Substituting (1) into (2) and integrating, $\Delta \mathrm{T}_{0}$ is obtained as;

$$
\Delta T_{0}=\frac{E}{\pi^{\frac{3}{2}} r_{0}{ }^{3} \rho_{\infty} c_{v}}
$$

In the study of Yan [9], the laser perturbation focal volume $V_{0}$ is $3 \mathrm{~mm}^{3}$ and is expressed as $V_{0}=(4 / 3) \pi R_{\mathrm{o}}{ }^{3} \cdot R_{\mathrm{o}}$ is assumed as the initial diameter and its value is $0.9 \mathrm{~mm} . r_{\mathrm{o}}$ is the initial radius and defined as $R_{\mathrm{o}} / 2$ and equals to $0.045 \mathrm{~mm}$.

This mathematical model was also validated by Yan $e t$ al.
[9] using Filtered Rayleigh Scattering method. The mathematical model of the laser pulse is used for the deposition of laser.

To understand the effect of laser energy on cavity flow, as it is given in Fig. 2, the pressure data are collected from cavity leading edge, cavity back wall and cavity floor.

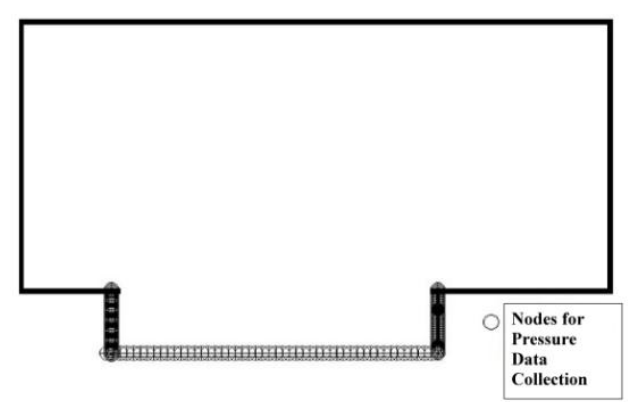

Fig. 2. Node locations for pressure data collection

The collected pressure data are used for the calculation of sound pressure level values (SPL). SPL distributions in the cavity region are considered as a comparison parameter for "with laser" and "without laser" cases. SPL values are calculated by the equations given below [1].

$$
\begin{gathered}
\bar{p}=\frac{1}{t_{f}-t_{i}} \int_{t_{i}}^{t_{f}} p d t \\
\bar{p}^{2}=\frac{1}{t_{f}-t_{i}} \int_{t_{i}}^{t_{f}}(p-\bar{p})^{2} d t \\
\mathrm{SPL}=10 \log _{10}\left(\frac{\bar{p}^{2}}{q^{2}}\right)
\end{gathered}
$$

$p$ is the pressure value at each node. $q$ is the reference sound pressure level value which is equal to $2 \times 10^{-5} \mathrm{~Pa}$. $\mathrm{t}_{\mathrm{i}}$ is the beginning time of data collection and $t_{f}$ is the end of collection [1].

\section{RESULT}

The CFD simulations show that pressure fluctuations become periodic after around 12 Rossiter periods and the laser energy deposition process starts after the flow become periodical as also reported by the study of Ayli [12] and Aradag et al. [10].To understand the impacts of laser energy deposition, different amounts of energy values are deposited to the flow just above the cavity leading edge which is represented with point 1 in Fig. 6. The laser energy deposition process starts at the instant that the flow becomes periodic and the laser energy is deposited to the flow from the same location at the beginning of each Rossiter period. One Rossiter period is 964 timesteps for this case (timestep: $10^{-6}$ s)as given in study of Ayli [12]. The CFD analysis is performed for 18 Rossiter periods, the first 12 is without laser, other 6 periods is with laser energy deposition. The SPL distributions are given in Fig. 3, Fig. 4 and Fig. 5.

As it is seen in Fig. 3, Fig. 4 and Fig. 5, for different amounts of laser energy values, SPL values which are 
related to pressure values do not change significantly. The differences are usually negligible.

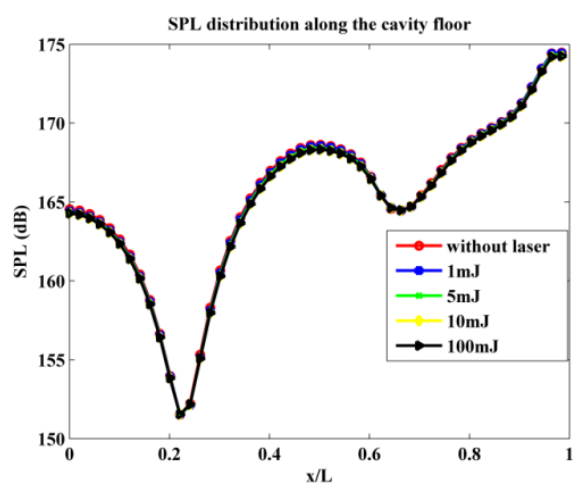

Fig. 3. SPL distribution along the cavity floor with and without laser

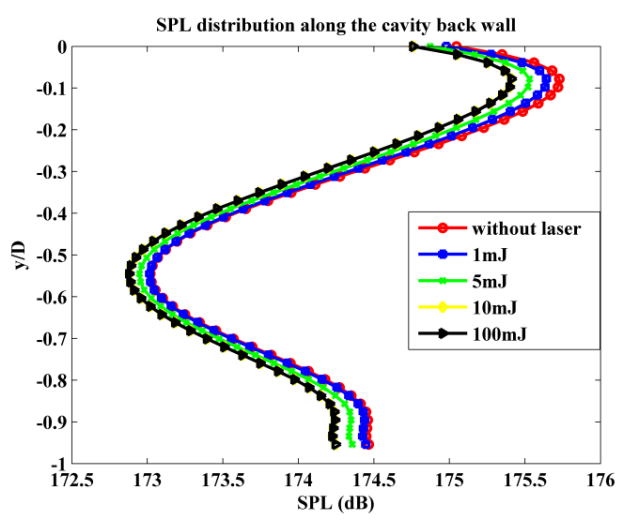

Fig. 4. SPL distribution along the cavity back wall with and without laser

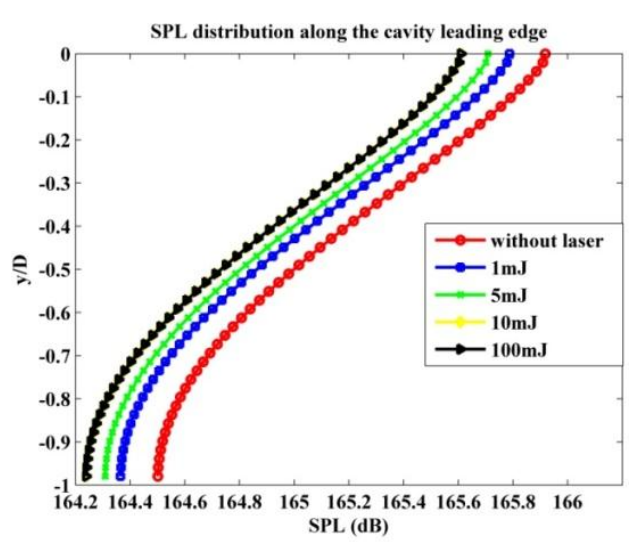

Fig. 5. SPL distribution along the cavity leading edge with and without laser

Other than the leading edge, two different locations are also studied for $100 \mathrm{~mJ}$ energy value. The locations that laser energy is deposited are given in Fig. 6.

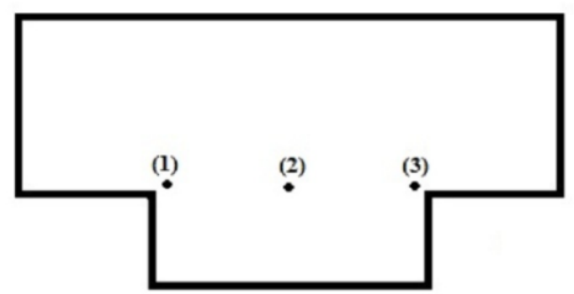

Fig. 6. Laser energy pulse locations

The highest SPL values occur at the back wall of the cavity. The critical pressure values are observed in this region. The SPL results at the cavity back wall for different locations of energy deposition are shown in Fig. 7.

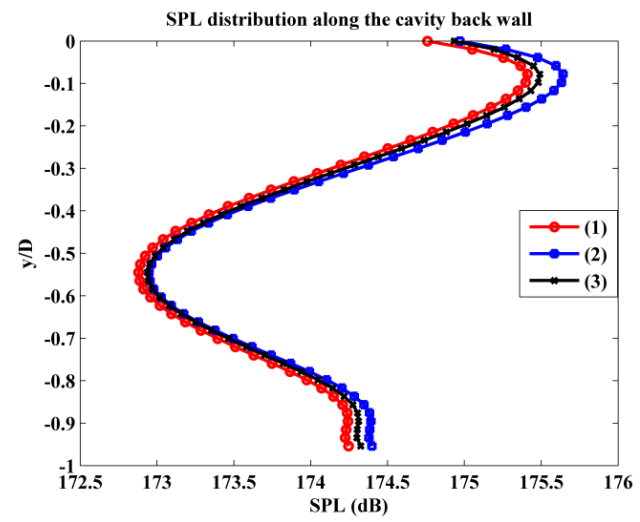

Fig. 7. SPL distribution along the cavity back wall for different locations of energy deposition, $\mathrm{E}=100 \mathrm{~mJ}$

As shown in Fig. 7, the results of the studied locations show no significant differences. The location just above the leading edge might be considered to be more efficient.

The effects of laser frequency are also studied. With the help of pressure contours taken along one Rossiter period, the laser pulse is examined. The pressure contours show that the propagation of laser pulse and its impacts disappear after a short time. Instead of providing laser energy only once per period, $100 \mathrm{~mJ}$ of laser energy is deposited to the cavity 10, 20, 30 and 40 times per period. For the frequency study, the laser energy deposition process is performed for just one period instead of six periods. The SPL distribution results are given in Fig. 8.

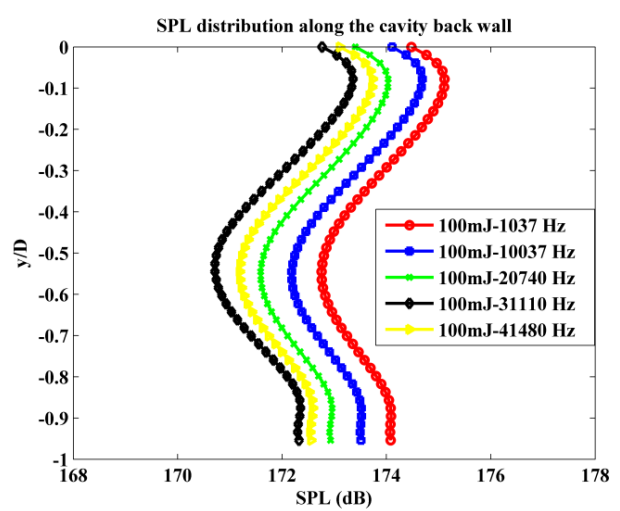

Fig. 8. SPL distribution along the cavity back wall for different frequencies, $\mathrm{E}=100 \mathrm{~mJ}$

Fig. 8 shows that the frequency value of $31110 \mathrm{~Hz}$, which is nondimensionalized dividing by Rossiter frequency of $1037 \mathrm{~Hz}$ (30 times per Rossiter period), is the most effective one. About $3 \mathrm{~dB}$ reduction is obtained in the SPL values at cavity back wall, when laser pulse is given to the cavity 30 times per fundamental Rossiter period.

\section{CONCLUSION}

There are several studies in literature about laser energy deposition to various types of flows to investigate its potential on flow control. The results of these studies show that laser energy may be effective for control purposes. In this study, the effects of laser energy on supersonic cavities are tested. The control parameters are the pressure distribution and relative sound pressure levels in the cavity region. 
The effects of location and frequency of laser energy deposition are investigated. $100 \mathrm{~mJ}$ of energy is deposited at three different locations in the cavity with a frequency of once per Rossiter period of the flow. The results did not show and significant impact. However, when the frequency is changed to 30 per Rossiter period a drop of $3 \mathrm{~dB}$ in sound pressure values is observed.

The preliminary results are promising. The effects of location, frequency and amount of laser energy deposition for supersonic cavities are still under investigation.

\section{ACKNOWLEDGMENT}

The authors would like to thank to Ece Ayli for her computational data for the supersonic cavity flow without energy deposition.

\section{REFERENCES}

[1] S. Aradag, CFD for High Speed Flows in Engineering, 1st ed., VDM Verlag Dr.Müller Altiengesellschaft and Co. Kg, Saarbrücken, Germany, 2008.

[2] D. L. Kral, Active Flow Control Technology, ASME Fluids Engineering Division Technical Brief, 1999.

[3] E. Lazar, G. Elliot, and N. Glumac, "Control of the Shear Layer Above a Supersonic Cavity Using Energy Deposition," AIAA Journal, vol.46, no.12, pp. 2987-2997, 1997.

[4] R. B. Miles, "Flow Control by Energy Addition into High-Speed Air," in Proc. AIAA Fluids Conference, Denver, 2000, AIAA Paper no. 2000-2324.

[5] R. Meyerand and A. Haught, "Gas break down at optical frequencies," Physical Review Letter, vol. 11, no. 9, pp. 401-403, 1963.

[6] E. Schülein, A. A. Zheltovodov, M. S. Loginov, and E. A. Pimonov, "Experimental and Numerical Study of Shock Wave Transformation by Laser- Induced Energy Deposition," in Proc. International
Conference on Methods of Aerophysical Research, ICMAR, Russia, 2008, Section II.

[7] S. H. Zaidi, M. N. Shneider, D. K. Mansfield, Y. Z. Ionikh, and R. B Miles, "Influence of upstream pulsed energy deposition on a shockwave structure in supersonic flow," in Proc. 22nd AIAA Aerodynamic Measurement Technology and Ground Testing Conference, St. Louis, Missouri, 2002, AIAA Paper no. 2002- 2703.

[8] R. Adelgren, G. Elliot, and D. Knight, "Laser energy deposition in transverse wall jets and intersecting shocks," in Proc. Second Workshop on Thermochemical Process in Plasma Aerodynamics, St. Petersburg, Russia, September 9-12, 2001.

[9] H. Yan, R. Adelgren, G. Elliott, D. Knight, T. Beutner, M. Ivanov, , A. Kudryavtsev, and D. Khotyanovsky, "Laser energy deposition in quiescent air and intersecting shocks," in Proc. Fourth Workshop on Magneto- and Plasma Aerodynamics for Aerospace Applications, IVTAN, Moscow, Russia, 2002.

[10] S. Aradag, H. Yan, and D. Knight, "The effects of laser energy deposition on supersonic cavity flow," Thermal Science and Technology, vol. 29, no. 2, pp. 67-73, 2009.

[11] E. Ayli, I. Yilmaz, and S. Aradag, "Numerical analysis of supersonic cavity flow," presented at the Sixth International Conference on Thermal Engineering Theory and Application, Istanbul, Turkey, May 29-June 1, 2012.

[12] E. Ayli, "Numerical analysis of supersonic cavity flow," M.S. thesis, Dept. Mechanical Eng., TOBB University of Economics and Technology, Ankara, Turkey, 2012.

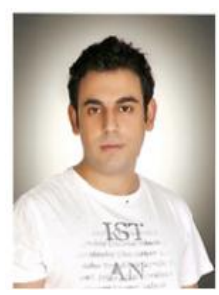

Ibrahim Yilmaz was born in Ankara. He graduated from Ankara Ataturk Anatolian High School in 2006. $\mathrm{He}$ graduated from TOBB University of Economics and Technology, Department of Mechanical Engineering in 2011. In the same year, he started his M.Sc. studies on computational fluid dynamics (CFD) and heat transfer in the same department. $\mathrm{He}$ is currently an M.S. student and a graduate research assistant in the same department. 